\section{Syntactic locus as a determinant of judged pause duration*}

\author{
SAMUEL FILLENBAUM \\ University of North Carolina, Chapel Hill, North Carolina 27514
}

This study sought to determine if the syntactic location of a speech pause might affect its apparent duration. Ss listened to a continuous discourse, being required to attend to its content as well as to make judgments, now and then, as to the duration of particular pauses. For pauses all actually of the same length, it was found that those falling at a minor within-sentence break, as syntactically defined, were characteristically judged to be longer than those falling at a major within-sentence break or at a between-sentence break, with no difference between the two latter cases. These results, obtained in a perceptual task involving a minimum of extraordinary or disruptive features, are taken as evidence for the power of syntactic variables in affecting attention during listening to speech.

In recent years there has accumulated considerable evidence that properties of the syntactic structure of a sentence may systematically affect the reported location of an extrinsic interrupting stimulus, such as a click heard while listening to speech. One important finding, for example, is that when listening to two-clause sentences Ss characteristically report a single intruding click in a location displaced toward the break between the two clauses (see, e.g., Fodor \& Bever, 1965; Bever, Lackner, \& Kirk, 1969). Since, in these experiments, Ss report on the location of the click after hearing and writing down the sentence, it is not at all clear that the effects are perceptual rather than memorial ones. In an attempt to clarify this matter, Bever and his associates have used GSR to shock (Bever, Kirk, \& Lackner, 1969) and immediate reaction time to a click (Abrams \& Bever, 1969) as more direct and immediate indices of S's response to speech as he hears it. While the results of these studies indicate that the syntactic structure of a sentence can systematically influence the GSR to shock and reaction time to clicks and that, therefore, syntactic knowledge may be used actively to modulate attention during listening, these studies also yield so many complex, and often unanticipated, findings and involve such a high ratio of inferences and hypotheses to data (see particularly Abrams \& Bever, 1969) as to leave matters rather unclear, and to raise the possibility that some of their results may be more directly attributable to special strategies used in coping with the unusual experimental situations, involving features disruptive of normal speech perception,

*This research was supported in part by USPHS Grant MH10006 from the National Institute of Mental Health. The writer is indebted to A. Horowitz for his aid in running $S s$ and analyzing the data. than to the normal processes the experiments sought to tap. structure on perceptual performance, it is clearly desirable that the task employed be a perceptual one and that the situation be as close as possible to the normal speech situation, involving a minimum of novel or possibly disruptive features. A task requiring the $S$ to assess the duration of some of the pauses in passages of continuous speech appears to meet these criteria in some measure; the task can reasonably be regarded as a perceptual or judgmental one. Speech is continuous, and by requiring Ss to provide a summary of be reasonably sure that he is attending to its content. There is no special intrusive stimulation to be dealt with, and, while the task of judging the duration of a pause now and then is certainly somewhat unusual, pauses do normally occur in speech, and often one is aware of them.

It is well known that the apparent or subjective duration of a time interval may be influenced by all sorts of variables (see, e.g., Fraisse, 1963; Frankenhaeuser, 1959). The specific concern of the present study is to determine if, and in what ways, the syntactic locus of a pause in speech may affect its apparent duration. One may distinguish between conventional or juncture pauses, which fall at syntactic boundaries, and idiosyncratic or hesitation pauses, which characteristically do not fall at such boundaries. Conventional pauses may permit the listener to cope with the analysis of completed phrases or clauses, while idiosyncratic pauses, which break up constituents, may make it harder for him to understand what is going on. Just because conventional pauses fall at breaks between major constituents, they may be less obtrusive and less salient, other things being equal, than pauses which fall within a major constituent, interrupting it. Indeed,
In investigating the effects of syntactic what has been said once in a while, one can as Garrett \& Fodor (1968, p. 462) observe in commenting on some findings of Boomer (1965) "....juncture pauses, which are linguistically determined, are not reported by observers as hesitation pauses in spite of the fact that such pauses are of significantly greater duration than pauses reported as hesitations. In fact such juncture pauses were long thought to be very much shorter than hesitation pauses." If a greater pause at a major syntactic boundary is less likely to be noticed than a lesser idiosyncratic or hesitation pause (see immediately above), then it would seem a fair inference that if a pause at a major syntactic break is precisely the same length as one that falls in the middle of nowhere (within a constituent), the former pause might appear to be of shorter duration. This was the hypothesis that guided the design of the present experiment where (in fact identical) pauses had to be judged, the pauses falling either (1) at a major syntactic break, which could be either (a) the break between two sentences or (b) a major within-sentence break, or (2) some place in the middle of nowhere within a constituent, at a minor within-sentence break. Previous work on this general issue, in particular the "click" research, has been concerned with the effects of intrasentence syntactic structure, and it is an interesting and open question as to whether the break between sentences will have a comparable, or even larger, effect than a major within-sentence break.

To summarize: we attempted to study the apparent duration of pauses in speech as a function of syntactic locus, using a perceptual task not too different from the normal speech-perception situation, seeking to determine whether a pause would be judged longer if it occurred within a major constituent than between constituents and if there would be any difference between results for the latter case and results for a pause occurring between sentences (thus extending this general paradigm from the study of within-sentence effects to the study of between-sentence effects).

Subjects

\section{METHOD}

The Ss were 67 undergraduates from an introductory psychology course who were fulfilling a class requirement for experimental participation. They were run in groups ranging in size from six or seven up to a dozen.

\section{Task and Procedure}

The materials, taken from the New York Times Magazine, consisted of an edited version of an interview with the then-Secretary of H.E.W., Wilbur Cohen. 
The format was a question-and-answer one, and the materials were recorded by an experienced student announcer, being read at a rather slow rate (about 120 words per minute). Occasionally a pause of exactly $2 \mathrm{sec}$ duration between words was then spliced into the tape. 1 These pauses fell in one of three locations: (1) at a between-sentence break, (2) at a major within-sentence break, most commonly between the two principal clauses of the sentence, and (3) at a minor within-sentence break, e.g., that between a modifier and its modified noun or between a determiner and its immediately following noun, etc. There were 120 such pauses in all, 40 of each kind, arranged in a haphazard sequence (in a text of somewhat less than 1,800 words).

The Ss were told that they would be listening to excerpts from an interview and that the experiment concerned their ability to do two things at the same time-to judge the length or duration of some of the pauses in the speaker's comments and to get the gist of these comments so as to be able to write down, immediately after each answer, the main points made. They were requested to make their judgments of pause duration in terms of a 5-point scale, ranging from 1 (very short) to 5 (very long), and were warned that the pauses would be very similar in length, "... that you will seldom have need to use either No. 1 or No. 5 but do your best to make the necessary fine discriminations by using the three middle categories." To indicate the pauses to be judged, the word immediately preceding each critical pause was provided on the response sheets. Ss were told that these words would signal that, in each case, the immediately following pause was to be judged as to duration and that they were, therefore, to keep an eye on these words while listening. Immediately after each answer by the Secretary of H.E.W., the tape was stopped, and, as they had been told, Ss were given about $1 \mathrm{~min}$ to write down the main points made in the reply. Before starting the experiment proper, a sample text was played to acquaint $S$ s with the procedure, and any questions about the task were answered.

\section{Scoring and Analysis of Data}

The early judgments made by the Ss were not scored, although they did not know that this would be the case, since we wished to permit them to become fully acquainted with the task and the pause durations (really duration) employed and for their judgment scales to become stabilized. Hence, we considered as data only the last 93 (out of 120) judgments made, 31 for each kind of pause location. ${ }^{2}$
For each $S$ the mean judgment made for each of these three classes of pauses was noted, and these values were then subjected to a rank test for correlated samples (Friedman test) and to sign tests.

There is a possible ambiguity with regard to the definition of major within-sentence breaks since, at the time they occur, these are sometimes potentially between-sentence breaks. Consider, e.g., the sentence, "He ate a large sandwich, because he was hungry." Listening to such a sentence, at the point immediately after "sandwich" one might be at the end of the sentence or, as is indeed true of the example, one might only be at the end of one major clause, with another clause yet to come. With these considerations in mind, the 31 major within-sentence breaks were reexamined and, in terms of syntactic considerations (and intonational information as appropriate), 10 cases were found which might have been regarded by Ss, at that point, as between-sentence breaks. These cases were reclassified, new means for each of the three classes of pauses for each $S$ were calculated (with those for major within-sentence breaks and between-sentence breaks now based on 21 and 41 cases, respectively), and the same analysis that was carried out earlier on the "raw" data was now performed on these "adjusted" data.

\section{RESULTS AND DISCUSSION}

Let us denote between-sentence pauses by $A$, major within-sentence pauses by $B$, and minor within-sentence pauses by $C$. For the "raw". data the means for these conditions are 2.96, 2.99, and 3.25, respectively; Friedman nonparametric analysis yields $\chi^{2}(2 \mathrm{df})=50.7, \mathrm{p}<.001$; contrasting the conditions by use of sign tests yields 53 cases where $\mathrm{C}>\mathrm{A}$ and 11 where $A>C, 55$ cases where $C>B$, and 9 where $\mathrm{B}>\mathrm{C}$, both of these contrasts being highly significant $(\mathrm{p}<.01)$, and 38 cases with $B>A$ and 24 with $A>B$ (n.s.). An analysis of the "adjusted" data yields means of 2.97, 2.98, and 3.25 for A, B, and $\mathrm{C}$, respectively; the Friedman test yields a $\chi^{2}(2 \mathrm{df})=43.4, \quad \mathrm{p}<.001$; contrasting conditions by use of sign tests yields 54 cases where $C>A$ and 11 where $A>C, 55$ cases where $C>B$ and 10 where $\mathrm{B}>\mathrm{C}$, both of these contrasts again being highly significant $(\mathrm{p}<.01)$, and 31 cases with $B>A$ and 33 with $A>B$ (n.s.). Obviously the results are just about the same whether "raw" or "adjusted" data are being considered, and reveal that $C$ is significantly greater than $A$ and than $B$ and that the latter two conditions do not differ from each other, i.e., under circumstances where all pauses are actually of the same duration, those falling at a minor within-sentence break are characteristically judged to be longer than those falling at a major within-sentence break or at a between-sentence break, the latter conditions yielding essentially the same results. ${ }^{3}$

These results indicate that under conditions where $S$ is required to carry out some syntactic (and semantic) analysis of the material he is listening to, so as to be able to report on its content, the apparent duration of a pause is conditioned by its syntactic locus, that a pause falling at a place where there is no syntactic reason for a pause (minor within-sentence break) is judged longer than a pause of the same length falling at some major break, where conventional or juncture pauses are syntactically appropriate, and that it make no difference whether such a break is a major within-sentence break or a between-sentence break. ${ }^{4}$ These findings of an effect of syntactic structure obtain for a task that can reasonably be characterized as a perceptual one, in a situation not too far removed from the normal speech situation, at least insofar as no potentially intrusive stimulus, such as a click or shock, is introduced. It should be noted, however, that in at least one respect studies that have used the latter sorts of techniques have an advantage over the present study, viz, that those studies have involved a richer syntactic analysis of pause locus than the three category scheme used in the present study, permitting their authors to make inferences with regard to the role of surface and deep structure breaks, particularly implicating the latter in the results (see, e.g., Bever, Lackner, \& Kirk, 1969).

There is not much in the way of data on perceived pause duration, as syntactically conditioned, with which the present findings might be compared directly. Our results are certainly consistent with expectations based on a consideration of Boomer's (1965) findings, noted earlier, indicating that juncture pauses which are often deemed very short are, in fact, significantly longer, on the average, than idiosyncratic hesitation pauses. If that is the case, then surely a pause which falls at a syntactic juncture and is of the same length as one that falls in the middle of a major constituent should be judged to be shorter than the latter, as was found here. Clark (in press) reports some preliminary findings where listeners were required to judge which of two $1 / 2$-sec pauses, variously located in two immediately successive sentences, seemed the longer. The pattern of results could not be related in any systematic way to the location of the pauses relative to phrase structure boundaries. In an unpublished monograph 
written in the middle 1960s, Fodor, Jenkins, and Saporta ${ }^{5}$ refer briefly to a then (and still now) unpublished study by Bever, indicating that the longest pause in a sentence is heard where the major constituent boundary falls. This result would appear to be exactly opposite to those obtained in the present study (and what would appear to be implied by Boomer's findings). In the absence of any further information on the Bever study, one cannot tell whether there is, indeed, a real conflict in results.

In some respects the results obtained in the present study are perhaps more impressive testimony to the power of syntactic properties than might appear at first consideration. On the assumption that, among other things, a listener must exercise his grammatical knowledge while listening and attempting to understand discourse, it may not be too surprising that pauses which fall at syntactically inappropriate locations are more obtrusive and more salient, and, therefore, appear to be longer than pauses which come at places where it is syntactically appropriate to pause. But note that this effect obtains in the present case under conditions where all of these variously located pauses are made equally obtrusive and salient by requiring $S$ to judge their durations. It might be of some interest to determine whether this effect would still obtain if $S$ were not required to attend to the content of the discourse in which the pauses are embedded, being urged to treat this as so much noise and to focus attention solely on the pause durations. If the effect were still to be found, it would provide strong evidence for the pervasive pull of syntactic variables, as though it were impossible to avoid syntactic processing of meaningful materials to which one is being exposed.

\section{REFERENCES}

ABRAMS, K., \& BEVER, T. G. Syntactic structure modifies attention during speech perception and recognition. Quarterly Joumal of Experimental Psychology, 1969, 21, 291-298.

BEVER, T. G., KIRK, R., \& LACKNER, J. R. An autonomic reflection of syntactic structure. Neuropsychologia, 1969, 7, 23-28. BEVER, T. G., LACKNER, J. R., \& KIRK, R. The underlying structures of sentences are the primary units in speech perception. Perception \& Psychophysics, 1969, 5, 225-234.

BOOMER, D. S. Hesitation and grammatical encoding. Language \& Speech, 1965, 8, 148-158.

CLARK, H. H. The importance of linguistics for the study of speech hesitations. In D. L. Horton and J. J. Jenkins (Eds.), The perception of language. In press.

FODOR, J. A., \& BEVER, T. G. The psychological reality of linguistic segments. Journal of Verbal Learning \& Verbal Behavior, 1965, 4, 414-420.

FRAISSE, P. The psychology of time. New York: Harper \& Row, 1963.

FRANKENHAEUSER, M. Estimation of time: An experimental study. Stockholm: Almqvist \& Wiksell, 1959.

GARRETT, M., \& FODOR, J. A. Psychological theories and linguistic constructs. In T. R. Dixon and D. L. Horton (Eds.), Verbal behavior and general behavior theory. Englewood Cliffs, N.J: Prentice-Hall, 1968. P. 596.

\section{NOTES}

1. The author wishes to express his thanks to Ron Cook of the University of North Carolina Recording Laboratory for his expert processing of these tapes.

2. More precisely, the data are based on 93 out of the last 96 judgments, since for three cases there was a possibility of confusion with regard to the identity of the critical pause; responses in these cases were not considered.

3. While not quite reaching significance, the data of an earlier study are consistent with those just presented. In the earlier study the $30 \mathrm{Ss}$ were run individually, and, instead of being provided with the word preceding each critical pause, each immediately impending pause was signaled by the winking on of a red light, while the word immediately preceding that pause was being played. The Friedman test yielded a $\chi^{2}(2 \mathrm{df})=5.27,(\mathrm{p} \cong .07)$; contrasting conditions by sign test yielded 19 cases where $C>A$ and 11 where $A>C, 20$ cases where $C>B$ and 8 where $B>C$, and 16 cases with $B>A$ and 10 with $\mathrm{A}>\mathrm{B}$; the means for $\mathrm{A}$ and $\mathrm{B}$ were identical, with that for $\mathrm{C}$ somewhat higher, as might be expected.

4. Obviously one must caution that these results may be specific to the particular pause interval employed, as embedded in speech at the particular given (slow) rate.

5. An introduction to psycholinguistic theory by J. A. Fodor, J. J. Jenkins, \& S. Saporta.

(Accepted for publication July 6, 1970.) 